\title{
Ddb1 controls genome stability and meiosis in fission yeast
}

\author{
Christian Holmberg, ${ }^{1,4}$ Oliver Fleck, ${ }^{1,2,4}$ Heidi A. Hansen, ${ }^{1,5}$ Cong Liu, ${ }^{3}$ Rita Slaaby, ${ }^{1,6}$ \\ Antony M. Carr, ${ }^{3}$ and Olaf Nielsen ${ }^{1,7}$ \\ ${ }^{1}$ Department of Genetics, Institute of Molecular Biology, University of Copenhagen, DK-1353 Copenhagen K, Denmark; \\ ${ }^{2}$ North West Cancer Research Fund Institute, University of Wales, Bangor, Gwynedd LL57 2UW, United Kingdom; ${ }^{3}$ Genome \\ Damage and Stability Centre, School of Life Sciences, University of Sussex, Falmer, Sussex BN1 9RQ, United Kingdom
}

The human UV-damaged DNA-binding protein Ddb1 associates with cullin 4 ubiquitin ligases implicated in nucleotide excision repair (NER). These complexes also contain the signalosome (CSN), but NER-relevant ubiquitination targets have not yet been identified. We report that fission yeast Ddb1, Cullin 4 (Pcu4), and CSN subunits Csn1 and Csn2 are required for degradation of the ribonucleotide reductase (RNR) inhibitor protein Spd1. Ddb1-deficient cells have $>\mathbf{2 0}$-fold increased spontaneous mutation rate. This is partly dependent on the error-prone translesion DNA polymerases. Spd1 deletion substantially reduced the mutation rate, suggesting that insufficient RNR activity accounts for $\sim 50 \%$ of observed mutations. Epistasis analysis indicated that Ddb1 contributed to mutation avoidance and tolerance to DNA damage in a pathway distinct from NER. Finally, we show that Ddb1/Csn1/Cullin 4-mediated Spd1 degradation becomes essential when cells differentiate into meiosis. These results suggest that Ddb1, along with Cullin 4 and the signalosome, constitute a major pathway controlling genome stability, repair, and differentiation via RNR regulation.

[Keywords: Genome stability; meiosis; ribonucleotide reductase; Ddb1; S. pombe]

Supplemental material is available at http://www.genesdev.org.

Received November 2, 2004; revised version accepted February 9, 2005.

Correct regulation of the availability of deoxynucleotide triphosphate (dNTP) is important for genome integrity. In budding yeast, each $S$ phase requires the activation of the ribonucleotide reductase (RNR) enzyme that catalyses dNTP formation (Huang and Elledge 1997; Huang et al. 1998). This in turn requires activation of the DNAintegrity-checkpoint pathways, and checkpoint mutants are inviable because they fail to degrade the RNR inhibitor Sml1 when cells are in S phase (Zhao et al. 1998). In response to genotoxic stress, genes encoding RNR are transcriptionally induced in a checkpoint-dependent manner. However, elevated nucleotide pools cause increased mutation rates in vivo (Chabes et al. 2003), suggesting that fine tuning of dNTP levels is important for the fidelity of DNA synthesis.

In Schizosaccharomyces pombe, the COP9/signalosome (CSN) is required for RNR activation prior to DNA synthesis and in response to DNA damage (Liu et al. 2003). Active RNR is a heterotetramer of two large

\footnotetext{
${ }^{4}$ These authors contributed equally to this work

Present addresses: ${ }^{5}$ Alpharma Aps, Dalslandsgade 11, DK-2300 Copenhagen S, Denmark; ${ }^{6}$ Novo-Nordisk A/S, 6B1.058, Novo Allé, DK-2880 Bagsværd, Denmark.

${ }^{7}$ Corresponding author.

E-MAIL onigen@my.molbio.ku.dk; FAX 45-35-322113.

Article and publication are at http://www.genesdev.org/cgi/doi/10.1101/ gad.329905.
}

(Cdc22) and two small (Suc22) subunits (Fernandez-Sarabia et al. 1993). Outside of S phase, RNR assembly is inhibited by a small protein Spd1, which anchors Suc22 in the nucleus, away from Cdc22 in the cytoplasm. When cells enter $S$ phase, Spdl gets ubiquitinated and degraded, and Suc22 is transported to the cytoplasm to form active complexes with Cdc22. Mutants lacking the signalosome subunits Csn1 or Csn2, or the Cullin 4 homolog Pcu4, fail to degrade Spd1 upon S-phase entry, and so Suc22 remains in the nucleus, causing slow S-phase progression and sensitivity to DNA-damaging agents (Liu et al. 2003).

The CSN is a conserved multiprotein complex required for proper development in multicellular organisms (Cope and Deshaies 2003). Genetic and biochemical evidence suggest CSN functions as both a positive and a negative regulator of Cullin-based ubiquitin ligases. CSN mutants are defective in Cullin-based ubiquitination (Cope and Deshaies 2003), suggesting a role in correct modulation of these activities. Conversely, purified CSN can cleave the stimulatory Nedd8 modification (termed neddylation) from Cullins (Lyapina et al. 2001). Fission yeast cells lacking CSN subunits accumulate Cullins in their active, neddylated state (Zhou et al. 2001; Mundt et al. 2002). Furthermore, both human and fission yeast CSN copurify with deubiquitinating activi- 
ties (Groisman et al. 2003; Zhou et al. 2003), suggesting CSN may also prevent Cullin-based ubiquitination under certain circumstances.

In fission yeast, the signalosome can be isolated as a complex, which also contains Cullin 4 (Pcu4) and Ddb1 (Liu et al. 2003). Ddb1 is the fission yeast ortholog of human p127 DDB1, which together with the WD-repeat protein p48-DDB2 forms a UV-damaged DNA-binding heterodimer (UV-DDB) implicated in nucleotide excision repair (NER) (see below). A second Ddb1-like protein, Rik1, seems to be present only in fission yeast and is required for heterochromatin formation and meiotic telomere clustering (Ekwall et al. 1996; Nakayama et al. 2001; Tuzon et al. 2004). The Ddb1 family of proteins contain putative RNA-binding regions (Neuwald and Poleksic 2000; Dichtl et al. 2002), but the function of these has not been defined. Direct sequence orthologs of Ddb1 and Cullin 4 are not present in Saccharomyces cerevisiae, suggesting that this entire pathway may have been lost or changed during evolution.

Two distinct, yet related, DDB1 complexes containing CSN and Cullin 4 were recently purified from human cells (Groisman et al. 2003). One contained DDB2, while the other contained another WD40-repeat protein CSA. DDB2 is mutated in xeroderma pigmentosum group $\mathrm{E}$ (XP-E) patients, who are hypersensitive to UV-light, predisposed to a range of cancers, and suffer defective global genomic NER (GG-NER) (see Wittschieben and Wood 2003), and defective UV-induced apoptosis (Itoh et al. 2003, 2004). Mutation of CSA causes Cockayne syndrome, a transcription-coupled (TC-) NER defect (for review, see Svejstrup 2002).

The dynamic behavior of the human DDB1 complexes is consistent with the idea that the CSN modulates Cullin-based ubiquitination. Upon UV-irradiation, the CSN leaves the DDB2 complex, which then becomes tightly associated with chromatin. At the same time, Cullin 4 becomes activated by neddylation and presumably ubiquitinates substrates important for GG-NER. Subsequently, CSN reassociates and Cullin 4 becomes deneddylated. siRNA knockdown of CSN subunit 5 significantly decreases GG-NER, suggesting a positive role for $\mathrm{CSN}$ in this process. Conversely, CSN is only recruited to the CSA complex following UV-irradiation, presumably inactivating Cullin 4, and suggesting that suppression of ubiquitination is important for the execution of TC-NER (Groisman et al. 2003). Taken together, these observations suggest that DDB1 functions to regulate protein ubiquitination, although the genetic evidence for this is still circumstantial.

Deletion of $S$. pombe $d d b 1$ has been reported to confer chromosome segregation defects, mild UV-sensitivity, and slow S-phase progression (Zolezzi et al. 2002; Bondar et al. 2003). Here, we show that fission yeast Ddb1 is required together with the signalosome and Pcu4 for degradation of Spd1 following DNA checkpoint activation. We demonstrate that $d d b 1$ loss is synthetically lethal with loss of the DNA-damage checkpoint genes $c d s 1$, chk1, and rad3, and that these colethalities are readily suppressed by deletion of spd1. Importantly, we find that $d d b 1$ and $\operatorname{csn} 1$ mutants have an increased spontaneous mutation rate, in part due to a deprived dNTP pool. Interestingly, the elevated mutation rates are also partially suppressed by inactivating translesion DNA polymerases. Finally, we demonstrate that mutants in $d d b 1$, $\operatorname{csn} 1$, and pcu 4 are completely unable to undergo meiotic differentiation, since these cells fail to enter premeiotic $S$ phase. This defect is suppressed by Spd1 loss, or alternatively, by Suc22 overexpression, consistent with the notion that these mutants suffer from insufficient levels of DNA building blocks.

\section{Results}

$\Delta \mathrm{ddb} 1$ cells have a mutator phenotype distinct from $N E R$ and $M M R$

Since human DDB1 has been implicated in NER, we tested the sensitivity to UV and methyl methane sulphonate (MMS) of fission yeast cells deleted for $d d b 1$ (Fig. 1). $\Delta d d b 1$ cells are mildly sensitive to UV and highly sensitive to MMS. In contrast, NER-deficient $\Delta$ swilo cells (lacking the ortholog of human ERCC1 excision nuclease (Rödel et al. 1992) are very sensitive to UV, but only modestly sensitive to MMS. Double-mutant $\Delta d d b 1$ $\Delta$ swi10 cells showed increased sensitivity to both UV and MMS when compared with either of the single mutants. These findings suggest that Ddb1 is important for the cellular responses to damaged DNA, but unlikely to have a significant function in NER.

We next tested whether Ddbl functions in mutation avoidance by measuring the spontaneous reversion rates of the ade6 alleles 485 and (GTCC) ${ }_{2}$-1399. The 485 allele reverts to $a_{d e}^{+}$via base substitution (Fleck et al. 1999), while (GTCC) ${ }_{2}-1399$ reverts by deletion or insertion of nucleotides (Marti et al. 2003b). The mutation rates of both ade6 alleles were increased $>20$-fold in $\Delta d d b 1 \mathrm{mu}-$ tants when compared with $d d b 1^{+}$(Table 1), suggesting that the function of Ddb1 is important for preventing mutations. NER and mismatch repair (MMR) are two of the important mutation-avoidance pathways in fission yeast (Rödel et al. 1992; Fleck et al. 1999; Rudolph et al. 1999). We thus examined the reversion rates of double mutants $\Delta d d b 1 \Delta s w i 10$ (NER) and $\Delta d d b 1 \Delta m s h 2$

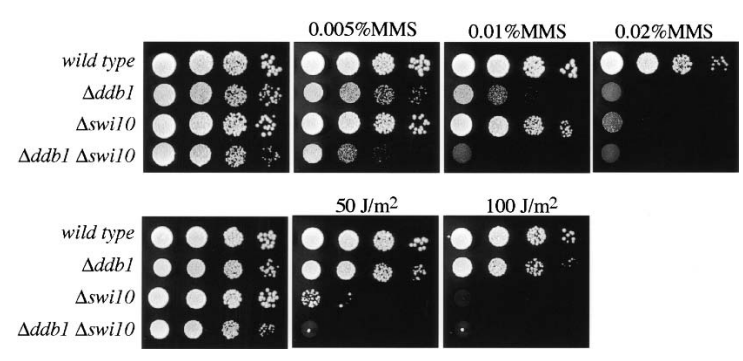

Figure 1. DNA-damage sensitivity of $\Delta d d b 1$ is nonepistatic to nucleotide excision repair. Strains of indicated genotypes were spotted in 10-fold dilutions and either left untreated (left), grown on MMS containing plates as indicated (top), or exposed to UV-C irradiation (bottom). 
Table 1. Mutation rates

\begin{tabular}{|c|c|c|c|c|}
\hline \multirow[b]{2}{*}{ Genotype } & \multicolumn{2}{|c|}{485} & \multicolumn{2}{|c|}{$(G T C C)_{2}$} \\
\hline & $\begin{array}{l}\text { Rate } \\
\left(\times 10^{-9}\right)\end{array}$ & Increase & $\begin{array}{c}\text { Rate } \\
\left(\times 10^{-10}\right)\end{array}$ & Increase \\
\hline wild type & $3.2(0.8)$ & 1 & $1.6(0.9)$ & 1 \\
\hline$\Delta d d b 1$ & $75(13)$ & 23 & $58(15)$ & 36 \\
\hline$\Delta m s h 2$ & $58(12)$ & 18 & $16(6.4)$ & 10 \\
\hline$\Delta s w i 10$ & $24(5.7)$ & 7.5 & 13 (9.9) & 8.1 \\
\hline$\Delta \operatorname{csn} 1$ & $18(3.6)$ & 5.6 & ND & \\
\hline$\Delta s p d 1$ & $2.0(1.2)$ & 0.6 & ND & \\
\hline$\Delta d d b 1 \Delta m s h 2$ & $252(54)$ & 79 & ND & \\
\hline$\Delta d d b 1 \Delta s w i 10$ & $146(40)$ & 46 & $183(28)$ & 114 \\
\hline$\Delta d d b 1 \Delta \operatorname{csn} 1$ & $69(35)$ & 22 & ND & \\
\hline$\Delta d d b 1 \Delta s p d 1$ & $37(15)$ & 12 & $15(4.0)$ & 9.4 \\
\hline$\Delta c s n 1 \Delta \operatorname{spd} 1$ & $7.6(1.3)$ & 2.4 & ND & \\
\hline$\Delta s w i 10 \Delta s p d 1$ & $21(4.8)$ & 6.6 & ND & \\
\hline$\Delta d d b 1 \Delta r e v 3$ & $39(3.1)$ & 12 & $40(9.1)$ & 25 \\
\hline$\Delta d d b 1 \Delta \operatorname{din} B$ & ND & & $33(14)$ & 21 \\
\hline$\Delta d d b 1 \Delta \operatorname{rev} 3 \Delta \operatorname{din} B$ & ND & & $21(11)$ & 13 \\
\hline$\Delta d d b 1 \Delta r e v 3 \Delta s p d 1$ & $24(6.3)$ & 7.5 & $27(4.1)$ & 17 \\
\hline
\end{tabular}

Reversion rates of the adeb alleles 485 and (GTCC) $)_{2}$ in the various backgrounds are mean values of at least three experiments with standard deviations in parenthesis (see Materials and Methods). Fold increases are relative to wild type. (ND) Not determined.

(MMR). Both exhibited higher mutation rates than either single mutant (Table 1), suggesting that Ddb1 does not prevent mutations by participating in MMR or NER.

We determined the mutation spectra of ade6-485 reversions in the various backgrounds. In wild-type cells, 485 reverted at about the same frequencies by G-to-C, G-to-T, A-to-G, or A-to-C base substitutions (Supplementary Table S1). In contrast, the $\Delta d d b 1$ mutant almost exclusively showed G-to-C and A-to-G events. This pattern is different from that of $\Delta$ swi10 and $\Delta m s h 2$, further supporting the idea that Ddb1 defines a novel mutation-avoidance pathway in fission yeast.

\section{Ddb1 is required for Spd1 degradation}

Ddb1 copurifies with the CSN and Pcu4, in a protein complex required for degradation of the RNR inhibitor Spd1 (Liu et al. 2003). This prompted us to test whether $\mathrm{Ddb} 1$ is required together with Csn 1 and Pcu4 for degradation of Spd1 in response to S-phase arrest. Figure 2A shows that $\Delta d d b 1$ cells-similar to $\Delta c s n 1$ and $\Delta p c u 4$ cells-are defective for Spd1 degradation following treatment with $20 \mathrm{mM}$ hydroxyurea (HU). Consistent with Ddb1 being required for Spd1 degradation, concomitant spd1 deletion improved the growth rates of $\Delta d d b 1$, $\Delta c s n 1$, and $\Delta p c u 4$ cells, and partially suppressed their elongated cellular appearance (data not shown). Flow cytometry for DNA content revealed a significant sharpening of the broad G2-peaks observed in $\Delta \operatorname{csn} 1$ and $\Delta d d b 1$ single mutants, suggesting that their slow S-phase progression was suppressed by deletion of $\operatorname{spd1}$ (Fig. 2B; Liu et al. 2003). In $\Delta p c u 4$, which shows the most severe phenotype of the three, concomitant spd1 deletion only marginally improved the flow cytometry profile. Thus, $\Delta$ pcu 4 cells suffer additional problems in cell cycle progression.

Since Spd1 is thought to inhibit RNR, and hence, to keep the deoxunucleotide pool low, we directly tested whether $\Delta d d b 1$ cells have reduced pools. We ${ }^{32} \mathrm{P}$-labeled asynchronously growing cells and extracted nucleotides in formic acid and resolved the individual nucleotide triphosphates by thin-layer chromatography. As shown in Supplementary Figure S1 and summarized in Table 2, the deoxy-pools are approximately threefold decreased in $\Delta d d b 1$, an effect that is suppressed by deletion of $s p d 1$. This relatively moderate reduction in pool sizes was expected, since only $\sim 10 \%$ of asynchronously growing cells are in $\mathrm{S}$ phase.

$\Delta \mathrm{ddb} 1$ is synthetically lethal with loss of the DNA-structure checkpoints in an spd $1^{+}$-dependent manner

Cells deleted for $\operatorname{csn} 1$ and $\operatorname{csn} 2$ are synthetically lethal with loss of any of the DNA-structure checkpoint genes rad3, cds1, and chk1, and this colethality is suppressed by overexpression of the small RNR subunit Suc22 (Mundt et al. 1999; Liu et al. 2003). The DNA-structure checkpoints are not essential under normal conditions (Caspari and Carr 1999), but become required for progression through $S$ phase when the availability of dNTP is limiting. To establish whether $\Delta d d b 1$ cells require DNA structure checkpoints for viability, we analyzed tetrads from crosses of $\Delta d d b 1$ with $\Delta r a d 3, \Delta c d s 1$, and $\Delta c h k 1$. In all three crosses, we failed to recover double-mutant colonies (Fig. 3), and double-mutant cells survived only a few divisions (data not shown). Thus, like $\operatorname{csn} 1$ and $\operatorname{csn} 2$ mutants, $\Delta d d b 1$ is colethal with loss of either the $\mathrm{S}$ phase or the G2 DNA-damage checkpoint. When we made the same crosses in spd1-deleted backgrounds, $\Delta d d b 1$ checkpoint double mutants were readily recovered (Fig. 3). We conclude that the inability to degrade
A

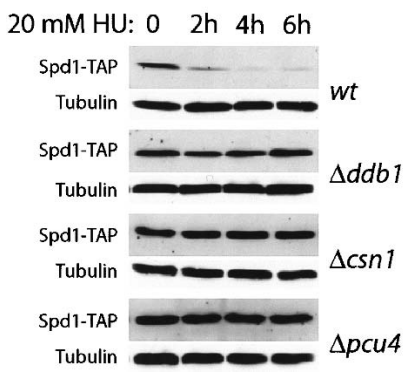

B

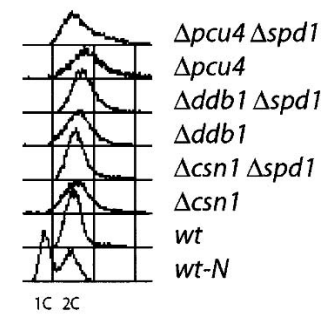

Figure 2. $\Delta d d b 1, \Delta c s n 1$, and $\Delta p c u 4$ are unable to degrade Spd1 in response to hydroxy-urea (HU). (A) TCA extracts of cells expressing Spd1-TAP tag and growing exponentially or treated with $20 \mathrm{mM}$ HU for 2, 4, and $6 \mathrm{~h}$ were subjected to Western blotting. (B) DNA content flow cytometry profiles of exponentially growing cultures as indicated. As control for G1 and G2 peaks (1C and 2C DNA content) the profile of wild-type cells starved for nitrogen for $6 \mathrm{~h}$ was included. 
Homberg et al.

Table 2. Nucleotide pools

\begin{tabular}{lcccc}
\hline & Wild type & $\Delta d d b 1$ & $\Delta d d b 1 \Delta s p d 1$ & $\Delta s p d 1$ \\
\hline ATP & 1 & 1 & 1 & 1 \\
GTP & 0.373 & 0.343 & 0.377 & 0.309 \\
CTP & 0.051 & 0.081 & 0.063 & 0.071 \\
UTP & 0.080 & 0.125 & 0.079 & 0.101 \\
dATP & 0.0156 & 0.0059 & 0.0180 & 0.0265 \\
dGTP & 0.0114 & 0.0037 & 0.0130 & 0.0169 \\
dCTP & ND & ND & ND & ND \\
dTTP & 0.0046 & 0.0016 & 0.0078 & 0.0135 \\
\hline
\end{tabular}

Relative amounts of the nucleotide triphosphates as normalized to the values obtained for ATP. dCTP has not been determined (ND), since we failed to detect signals above background for this nucleotide. The results have been reproduced in an independent experiment.

Spd1 makes $\Delta d d b 1$ cells strictly dependent on intact DNA structure checkpoints. Consistent with this, we found that cells without Ddb1 have elevated Cds1 kinase activity (data not shown), as also reported previously (Bondar et al. 2003).

spd1 deletion partially suppresses sensitivity to DNA damage in $\Delta \mathrm{ddb} 1$ and $\Delta \mathrm{csn} 1$

Since loss of spd1 largely suppressed the slow S-phase profile and damage sensitivity of $\Delta \operatorname{csn} 1$ (Liu et al. 2003) and the DNA structure checkpoint dependency of the $\Delta d d b 1$ mutant (this study), we speculated that spd1 loss might also affect the DNA damage sensitivity of $\Delta d d b 1$. In Figure 4A we show that the mild UV sensitivity of $\Delta d d b 1$ and $\Delta c s n 1$ cells at a relatively high dose of 160 $\mathrm{J} / \mathrm{m}^{2}$ was almost completely suppressed by deletion of spd1. In contrast, the UV sensitivity of NER-defective $\Delta$ swil0 cells was not suppressed by spd1 inactivation (Supplementary Fig. S3). Thus, the inability to activate RNR by Spd1 degradation upon severe UV damage causes lethal events at an elevated rate, at least partly due to lack of dNTP for DNA synthesis during repair and replication. In contrast, pcu4-deleted cells were more sensitive than $\Delta d d b 1$ and $\Delta c s n 1$, and their sensitivity was not suppressed by spd1 loss, suggesting that Pcu4 controls additional aspects of the UV-damage response.

MMS treatment causes a variety of lesions, including DNA-strand breaks, and all three mutants were found to be highly sensitive to this drug (Fig. 4B); $\Delta$ pcu4 cells showed the strongest sensitivity and, again, this was only marginally suppressed by lack of Spd1. Cells deleted for $d d b 1$ were slightly less sensitive than $\Delta p c u 4$, and elimination of Spd1 significantly suppressed this at the lower dose used (0.005\% MMS), whereas less suppression was observed at $0.01 \%$ MMS. Finally, csn1-deleted cells were the least sensitive of the three, and here, we observed the best suppression by spd1 deletion. Analysis of the double-mutant $\Delta d d b 1 \Delta c \operatorname{sn} 1$ indicated that $d d b 1$ and $\operatorname{csn} 1$ belong to the same epistasis group with respect to MMS sensitivity (Fig. 4B). Taken together, these observations suggest that Ddb1/Pcu4/Csn1-mediated deg- radation of Spd1 is important in response to moderate levels of DNA damage. After more severe damage, other Ddb1/Pcu4-mediated reactions that do not require the signalosome become necessary.

\section{Lack of Spd1 partially suppresses the mutator phenotype}

Given these results, we next asked whether the mutator phenotype of $\Delta d d b 1$ cells also involves inability to degrade Spd1. First, we tested whether Csn1 was part of this mutation-avoidance pathway. Indeed, $\Delta \operatorname{csn} 1$ cells showed a significantly increased reversion rate of ade6485 , albeit not as high as that observed in $\Delta d d b 1$ cells (Table 1). The $\Delta d d b 1 \Delta c s n 1$ mutant had about the same reversion rate of ade6-485 as $\Delta d d b 1$, suggesting an epistatic relationship between $\Delta d d b 1$ and $\Delta c s n 1$, also in mutation avoidance. However, since mutation rates of $\Delta d d b 1$ cells were significantly higher than that of $\Delta c \operatorname{cs} 1$, Ddb1 likely contributes to genomic stability, also through mechanisms that do not depend on the CSN. Due to their very poor plating efficiency, we have been unable to measure mutation rates in $\Delta$ pcu 4 cells.

We reasoned that the inability of $\Delta d d b 1$ and $\Delta c s n 1$ cells to degrade Spd1 might create mutations via repli-

A
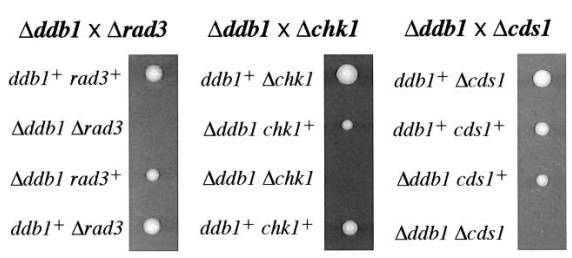

B

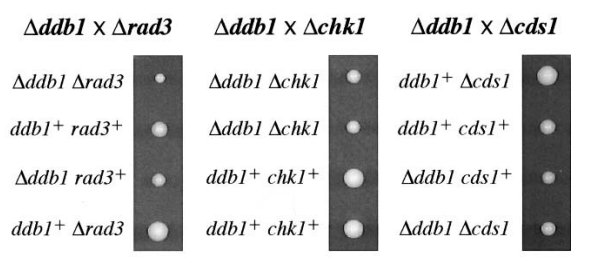

C

\begin{tabular}{|c|c|c|}
\hline Cross & TT & NPD \\
\hline$\Delta d d b 1 \times \Delta r a d 3$ & 4 & 0 \\
$\Delta d d b 1 \times \Delta c h k 1$ & 7 & 0 \\
$\Delta d d b 1 \times \Delta c d s 1$ & 4 & 1 \\
$\Delta d d b 1 \Delta s p d 1 \times \Delta r a d 3 \Delta s p d 1$ & 6 & 2 \\
$\Delta d d b 1 \Delta s p d 1 \times \Delta c h k 1 \Delta s p d 1$ & 5 & 3 \\
$\Delta d d b 1 \Delta s p d 1 \times \Delta c d s 1 \Delta s p d 1$ & 4 & 4 \\
\hline
\end{tabular}

Figure 3. Deletion of spd1 suppresses synthetic lethality of $\Delta d d b 1$ with loss of the DNA-damage checkpoint. $(A)$ Examples of tetratype tetrads of the crosses indicated. Genotypes are indicated at left, and they demonstrate that coloss of $d d b 1$ and the DNA-damage checkpoint is lethal. (B) As in $A$, except all mutants used were also deleted for spd1. (C) Table summarizing the number of tetrads analyzed from the crosses indicated at left, all with the outcome that deletion of $d d b 1$ is colethal with deletion of rad3, chk1, and cds1 in a manner suppressed by deletion of spd1. (TT) Meiotic segregation of the mutant alleles into tetra-type asci, i.e., all four spores have different genotypes; (NPD) nonparental ditypes, i.e., the mutant alleles cosegregate. 
A
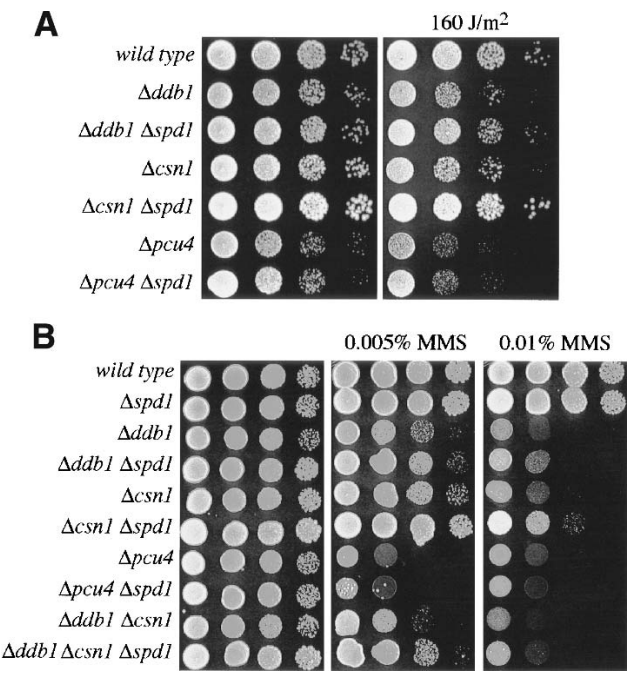

Figure 4. Deletion of $s p d 1$ suppresses sensitivity of $\Delta d d b 1$ and of $\Delta \operatorname{csn} 1$ to DNA damage. Survival tests of 10 -fold dilution series of the indicated strains. $(A)$ Right after spotting, plates were either left unirradiated or exposed to $160 \mathrm{~J} / \mathrm{m}^{2}$ of UV-C light. $(B)$ The strains were spotted onto plates containing $0 \%, 0.005 \%$, or $0.01 \%$ MMS as shown.

cation errors caused by insufficient dNTP pools. Consistent with this idea, we found that the ade6-485 reversion rates of the $\Delta d d b 1$ and $\Delta c s n 1$ mutants were reduced by $\sim 50 \%$ by deletion of the spd1 gene (Table 1). However, with significant mutator activity left in both double mutants, most notably in $\Delta d d b 1 \Delta s p d 1$, it is clear that Ddb1 and Csn1 are required for other aspects of mutation avoidance than for controlling RNR activation through Spdl degradation.

\section{Involvement of translesion DNA synthesis}

We next tested whether translesion synthesis (TLS) was involved in the increased mutation rate of $\Delta d d b 1$ cells. Unlike the replicative DNA polymerases, TLS polymerases can bypass certain types of lesions in DNA, but in doing so, they often incorporate erroneous nucleotides, thereby creating mutations (Friedberg et al. 2002; Hubscher et al. 2002). We tested the effects of mutating rev3, encoding the catalytic subunit of PolZ and $\operatorname{din} B$, coding for the Y-polymerase PolK (Wang 2001; Friedberg et al. 2002; Lehmann 2002; Kai and Wang 2003). Inactivation of one or both TLS polymerases partially suppressed the mutator phenotype of $\Delta d d b 1$ by $\sim 50 \%$ (Table 1 ). Thus, a considerable proportion of mutational events in $\Delta d d b 1$ cells depend on active TLS. Interestingly, mutation rates in the triple-mutant $\Delta d d b 1 \Delta$ rev3 $\Delta s p d 1$ were not, or only slightly further reduced when compared with the double-mutants $\Delta d d b 1 \Delta s p d 1$ and $\Delta d d b 1 \Delta r e v 3$, suggesting an epistatic relationship between the $\Delta s p d 1$ and $\Delta$ rev3 mutants in this context. In contrast to $\Delta d d b 1$ cells, the mutator phenotype of NER-defective $\Delta$ swi10 cells was unaffected by spd1 inactivation (Table 1), but is suppressed to wild-type level in the $\Delta r e v 3$ background (Marti et al. 2003a).
In the $(G T C C)_{2}$ reversion assay, the $\Delta d d b 1$ mutant exhibited a mutational hotspot of $\mathrm{C}_{2}$ to $\mathrm{C}_{1}$ 1-bp deletions, as well as some complex events, both of which were not found in the wild type (Supplementary Fig. S2). The complex events represented deletion or insertion of nucleotides accompanied by one or more base substitutions in the vicinity. Such events were not observed in the double-mutants $\Delta d d b 1 \Delta r e v 3$ and $\Delta d d b 1 \Delta \operatorname{din} B$ or the triple mutant $\Delta d d b 1 \Delta \operatorname{din} B \Delta r e v 3$, suggesting that they were due to error-prone TLS opposite spontaneous damage. However, the complex events were still observed in the $\Delta d d b 1 \Delta s p d 1$ strain, again consistent with Ddbl being required for other aspects of mutation avoidance than Spdl degradation.

The $\mathrm{C}_{2}$ to $\mathrm{C}_{1}$ hotspot remained in the $\Delta d d b 1$ double mutants with concomitant deletion of rev3, $\operatorname{din} B$, or spd1 (Supplementary Fig. S2). However, when considering the mutation rates, deletion of spd1 clearly reduced their occurrence, while no major effect could be seen by deleting $\Delta$ rev3 and/or $\Delta \operatorname{din} B$ (Supplementary Table 2). Taken together, these results suggest that Ddb1 loss causes mutations both by Spd1-dependent and independent mechanisms; for both types translesion polymerases can be involved.

\section{Spd1 degradation is required for entry into meiosis}

So far in this report, we have focused on the damage/ repair aspects of the Ddb1/Pcu4/Csn1 complex. However, CSN has been implicated in several developmental processes, and it has been proposed that its activity is crucial when cells must rapidly modify their physiology in response to signals received from the environment (Wei and Deng 2003). We therefore examined whether the Ddbl pathway was required when $S$. pombe cells undergo sexual differentiation. In response to nitrogen starvation, fission yeast cells first conjugate to form diploid zygotes, which then undergo meiosis and sporulation (Nielsen 2004; Yamamoto 2004). While homothallic $h^{90}$ strains deleted for $\operatorname{csn} 1, d d b 1$, or pcu 4 did conjugate, we found that the resulting zygotes were completely unable to undergo meiosis (Fig. 5; Table 3). Genetic analysis revealed that only $\operatorname{csn} 1$ and $\operatorname{csn} 2$ were required for meiosis, while $\operatorname{csn} 3-\operatorname{csn} 7$ were not (data not shown). Thus, the same two CSN subunits that control Spdl degradation are also required for entry into meiosis.

We next investigated whether Spdl loss could restore meiosis in these mutant cells. As shown in Figure 5 and Table 3, ddb1, csn1, and pcu 4 mutant strains readily underwent meiosis and sporulation when the spd1 gene had been deleted. Suc22 overproduction also suppressed the meiotic defects of these mutants (Table 3). The restored meiosis was phenotypically normal with wildtype recombination frequencies (Supplementary Fig. S4). Taken together, these findings show that Csn1, Csn2, Ddb1, and Pcu4 all function in the same pathway to liberate Suc22 from Spd1 repression during meiotic differentiation.

To identify where meiosis was blocked, we developed a meiotic synchronization procedure based on the obser- 


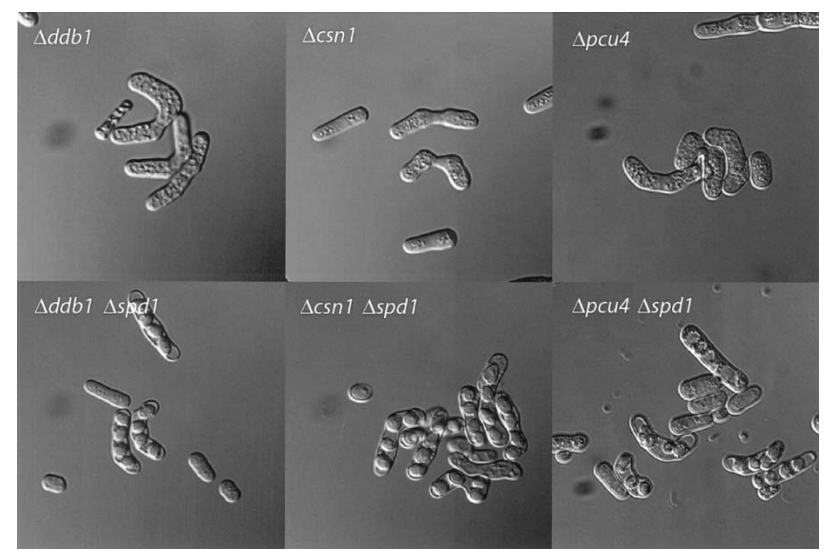

Figure 5. Deletion of $s p d 1$ suppresses the complete meiotic failure of $\Delta d d b 1, \Delta c s n 1$, and $\Delta p c u 4$. Microphotographs of $h^{90}$ mutant strains as indicated after $4 \mathrm{~d}$ on plates inducing mating and meiotic differentiation.

vation that stable diploid $h^{+} / h^{-}$cells mutated in the mat1-Mc gene require $\mathrm{M}$-factor pheromone in order to enter meiosis (Willer et al. 1995). When a growing culture of such cells was transferred to nitrogen-free medium containing $\mathrm{M}$-factor, cells first rapidly accelerated into mitosis consistent with earlier reports (Fantes and Nurse 1977; Stern and Nurse 1997). This gave rise to a transient accumulation of G1 cells, which then directly entered meiosis. Following this procedure, $>95 \%$ of wildtype cells had completed premeiotic $S$ phase after $5 \mathrm{~h}$ (Fig. 6A, first row). If entry into meiosis was blocked by the mat1-Mm mutation (Willer et al. 1995), all of the cells arrested in G1 (Fig. 6B, second row), showing that they could no longer enter mitotic $S$ phase.

Analysis of diploid $\Delta d d b 1 / \Delta d d b 1$ cells by this procedure was obscured by their slow $S$ phase, reflected in very broad flow cytometry profiles of DNA content (Fig. $2 \mathrm{~B}$; data not shown). We therefore studied the meiotic block in a $\Delta \operatorname{csn} 1$ mutant background, where the DNA profile is less severely affected. Diploid $\Delta \operatorname{csn} 1 / \Delta \operatorname{csn} 1$ cells still responded to the meiotic conditions by accumulating in G1, but little or no entry into premeiotic DNA synthesis was apparent during the course of the experiment (Fig. 6A, third row). Mutating spd1 or overproducing Suc22 restored entry into premeiotic $S$ phase with almost wild-type kinetics in both $\Delta \operatorname{csn} 1 \Delta / \operatorname{csn} 1$ and $\Delta d d b 1 / \Delta d d b 1$ (Fig. 6A; data not shown).

Next, we looked at Spd1 protein levels in cells induced to undergo meiosis. Spd1 was barely detectable in growing wild-type cells, but its level increased during the first $2 \mathrm{~h}$ following the shift to meiotic conditions, only to decrease again as the cells entered premeiotic $S$ phase (Fig. 6B). This pattern suggests that premeiotic S phasesimilar to mitotic $\mathrm{S}$ phase (Liu et al. 2003)-is accompanied by degradation of the Spd1 protein. In the $\Delta \operatorname{csn} 1 /$ $\Delta c s n 1$ cells, the level of Spd1 was not reduced; rather, it gradually increased during the meiotic conditions. In cells suppressed for the meiotic defect by Suc22 overexpression, the basal Spdl content was relatively high and stayed high during meiotic progression, suggesting the existence of a positive feedback between high Suc22 and Spd1. In $\Delta d d b 1 / \Delta d d b 1$ diploids, the level of Spd1 was higher than in $\Delta \operatorname{csn} 1 / \Delta \operatorname{csn} 1$ and stayed high during meiotic conditions, in agreement with the meiotic block being caused by failure to degrade Spd1.

To compare directly the level of Spd1 in the different strains, we ran the 5-h sample from each experiment on the same gel (Fig. 6C). This confirmed that $\Delta \operatorname{csn} 1 / \Delta \operatorname{csn} 1$ and $\Delta d d b 1 / \Delta d d b 1$ diploid cells arrest before premeiotic $S$ phase with elevated levels of Spd1 protein. We conclude that $\Delta \operatorname{csn} 1 / \Delta \operatorname{csn} 1$ and $\Delta d d b 1 / \Delta d d b 1$ mutant cells arrest before or in early premeiotic $S$ phase, due to defects in down-regulation of Spd1. Hence, meiotic DNA replication is absolutely reliant on the ability to degrade the RNR inhibitor Spd1 by a Ddb1-dependent process.

\section{Discussion}

The human p127 DDB1 protein has attracted considerable interest because of its implication in DNA repair reactions, and recently, DDB1 was shown to be a central component of two related Cullin 4-based E3 ubiquitin ligases (Groisman et al. 2003). One complex contained the DDB2 protein, which is defective in patients suffering from the GG-NER disease XP-E, while the other contained CSA, a protein function lacking in patients with the TC-NER disease CS. Hence, Cullin 4-mediated ubiquitination reactions seem important for the execution of nucleotide excision repair, but no substrates for these reactions have yet been identified. Both E3 complexes also contain the COP9/signalosome, suggesting that the CSN may modulate the spatial and/or temporal activity of these ligases.

\section{Spd1 as a major target for Ddb1}

In the present study, we have examined the function of fission yeast Ddb1. We provide evidence that in this system, the protein also collaborates with the Cullin 4 homolog Pcu 4 and the CSN in ubiquitin-mediated protein degradation, suggesting an evolutionary conservation of this pathway. We have identified the RNR inhibitor protein Spd1 as a major critical target. All of the phenotypes

Table 3. Frequency of spore containing zygotes (\%)

\begin{tabular}{lrr}
\hline$h^{90}$ strains & $2 \mathrm{~d}$ & $4 \mathrm{~d}$ \\
\hline wild type & 74 & 97 \\
$\Delta d d b 1$ & 0 & 0 \\
$\Delta d d b 1$ sspd1 & 62 & 84 \\
$\Delta d d b 1$ adh-suc22 & 47 & 82 \\
$\Delta \operatorname{csn} 1$ & 0 & 0 \\
$\Delta \operatorname{csn} 1 \Delta \operatorname{spd} 1$ & 66 & 75 \\
$\Delta \operatorname{csn} 1$ adh-suc22 & 54 & 76 \\
$\Delta p c u 4$ & 0 & 0 \\
$\Delta p c u 14 \operatorname{spd} 1$ & 21 & 46 \\
$\Delta \operatorname{csn} 5$ & 72 & 81
\end{tabular}

Percentage of zygotes developed into asci after 2 or $4 \mathrm{~d}$ incubation on plates supporting mating and meiosis. Genotypes are indicated on the left side. 
A

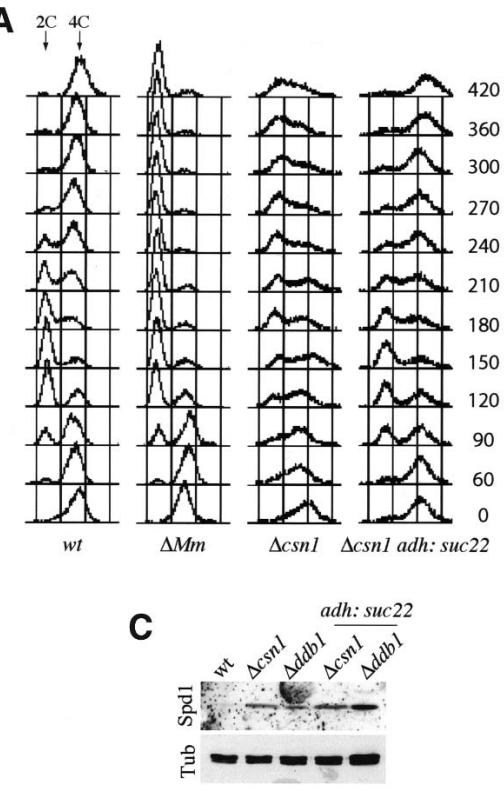

B

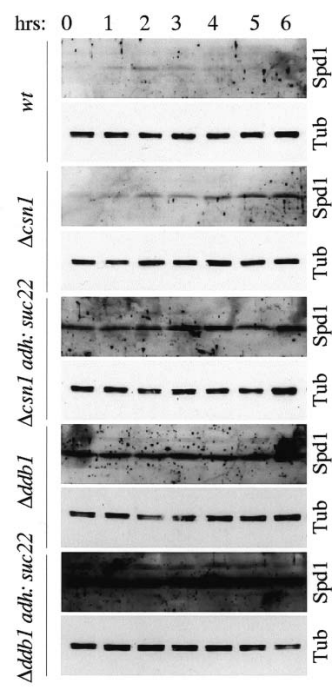

Figure 6. Pre-meiotic S-phase defect correlates with failure to down-regulate Spd1. (A) DNA content profiles of diploids as indicated shifted to meiotic conditions at time 0 with samples taken up to $420 \mathrm{~min}$ as shown at right of the profiles. $(B)$ Western blots for Spd1 in the diploids induced to undergo meiosis with samples taken every hour up to $6 \mathrm{~h}$. Anti-tubulin blots were included as loading control. Blots were run and developed in parallel to ensure direct comparability. $(C)$ Western blot for Spd1 using the 6-h time-point samples from $B$ to demonstrate on the same gel the higher levels of Spd1 in the $\Delta d d b 1 / \Delta d d b 1$ and $\Delta \operatorname{csn} 1 / \Delta \operatorname{csn} 1$ strains.

we have found associated with Ddb1 loss can, to a certain extent, be suppressed by deletion of spd1.

Our results demonstrate that Ddb1/Pcu4/Csn1-mediated degradation of Spd1 defines a new damage-response pathway important for genome integrity in fission yeast. Failure to degrade Spd1 causes slow S-phase progression, sensitivity to DNA-damaging agents, and increased mutation rates. In addition, survival of unperturbed cells becomes reliant on intact DNA structure checkpoints. Finally, the ability to degrade Spd1 is absolutely necessary when cells switch to the alternative developmental program of meiosis. This result suggests that meiotic $S$ phase is more sensitive to Spd1-mediated RNR inhibition than mitotic $S$ phase, which does proceed, albeit more slowly than normal. Consistent with this, it was previously reported that the $c d c 22-45$ mutant carrying a temperature-sensitive allele in the gene for the large RNR subunit prevented premeiotic $S$ phase at intermediate temperatures that still allowed mitotic $S$ phase (Grallert and Sipiczki 1991).

Our observations suggest that the phenotypes associated with defective Spd1 degradation during DNA replication and repair are due to insufficient dNTPs levels. Thus, meiosis could be completely restored in diploid $d d b 1 / d d b 1$ cells by overexpression of the small RNR subunit Suc22. Similarly, the radiation sensitivity and dependency on checkpoints of $\operatorname{csn} 1$ cells could be rescued by Suc22 overexpression (Liu et al. 2003). Our measurements confirmed that $\Delta d d b 1$ cells have reduced dNTP pools, and that deletion of spd1 could restore the pools above wild-type levels.

The involvement of Ddb1 in Spd1 degradation was anticipated from the observation that Ddb1 copurifies with the Pcu4/Csn complex (Liu et al. 2003), and a recent study has reported the requirement for Ddb1 in this process (Bondar et al. 2004). However, the same group arrived at a conclusion entirely different from ours regard- ing the relationship between Ddb1 and the DNA structure checkpoint pathway. Whereas we found that $\Delta d d b 1$ cells die in an Spd1-dependent manner in the absence of Rad3, Chk1, or Cds1, Bondar et al. (2003) reported that Cds1 loss suppressed the slow S-phase progression and sensitivity to DNA damage of $\Delta d d b 1$ cells. We do not know the basis for this discrepancy.

Although Spd1 clearly is a key substrate for the Ddb1/ Pcu4 pathway, it is evident from the incomplete suppression by spd1 loss we observe-especially of the mutator phenotype and the MMS sensitivity-that other targets exist. Recently, it was shown that Cullin 4 prevents rereplication in Caenorhabditis elegans by ubiquitination of the licensing protein Cdt1 (Zhong et al. 2003). Furthermore, in both HeLa and Drosophila cells, Cullin 4, Ddb1, and the CSN are required for degradation of Cdt1 following irradiation (Higa et al. 2003; Hu et al. 2004). Thus, the picture emerging is that Cullin 4-mediated ubiquitination reactions play an important role in maintenance of genome integrity at the G1-S transition and during repair. In budding yeast, where direct sequence orthologs of Ddb1 and Cullin 4 are absent, the checkpoint protein Rad53 controls RNR activity. It is possible, though, that less-obvious orthologs exist in this yeast to maintain vestigial Ddb1/Cullin 4-like functions, e.g., mutation of a cullin-like encoding gene rtt101 confers increased MMS sensitivity (Laplaza et al. 2004).

Human DDB1 was initially identified, together with the WD-40 repeat protein 448 DDB2, as an activity that associates tightly to UV-damaged DNA. Subsequently, NER-deficient XP-E patients were shown to be unable to form this complex, due to mutational inactivation of the DDB2 gene (for review, see Tang and Chu 2002). The role of UV-DDB in NER is unclear. Albeit not directly required for reconstituted NER on naked DNA in vitro, UV-DDB does stimulate NER in such an assay (Aboussekhra et al. 1995; Mu et al. 1995). It has been 
proposed that UV-DDB may function to assist the NER complex in gaining access to lesions in chromatin (Tang and Chu 2002), but other studies have shown that DDB1/DDB2 does not stimulate excision from nucleosomal DNA in vitro (Hara et al. 2000). The S. pombe genome contains no apparent DDB2 ortholog, and our genetic results suggest that Ddb1 functions in an epistasis group different from NER with respect to DNA-damage sensitivity and spontaneous mutation rates.

Fine tuning of dNTP levels is important for genome stability

We have provided evidence that $\Delta d d b 1$ cells have a strong mutator phenotype. The reversions we observed include both base substitutions and small insertion/deletions at highly increased frequency. As spd1 deletion suppressed the reversion frequencies to approximately half, our results suggest that a reduced nucleotide pool may be directly mutagenic. The fact that spd1 deletion appeared largely epistatic with inactivation of the DNA translesion polymerase PolZ in the mutation analysis, indicates that insufficient availability of dNTPs may favor recruitment of error-prone TLS polymerases, perhaps to extend stalled forks of the normal replicative polymerases. Indeed, it has been shown previously that replicational stress causing checkpoint activation promotes mutagenic translesion synthesis (Kai and Wang 2003). The increased dNTP pools in the spd1 background (Table 2) may be sufficient to circumvent checkpoint activation and the recruitment of translesion synthesis; hence, additional deletion of rev3 would reduce the mutation rate only a little further. This model is in agreement with our observation that viability of $\Delta d d b 1$ cells is dependent on the checkpoint kinases only in the presence of Spd1.

It will be interesting to learn whether the Ddb1 pathway also contributes to genome stability via activation of RNR in other systems like human cells. In budding yeast, it was-oppositely-demonstrated that an elevated dNTP pool could be mutagenic /Chabes et al. 2003), emphasizing the importance of proper RNR regulation for maintenance of genomic integrity. Our results may be important in cancer biology. Many cancer cells have constitutively high RNR activity due to inactivation of the pRB pathway (Angus et al. 2002), but we hypothesise that other cancer cells may adapt to a mutator strategy involving down-regulation of RNR and, hence, a slow, mutagenic S phase. Indeed, despite clear oncogenic effects of RNR overexpression, recent studies suggest that compromised RNR activity may also be common in human cancers, as the gene encoding the large RNR subunit $\mathrm{R} 1$ is located in the $11 \mathrm{p} 15.5$ region, which frequently displays one-allelic loss in solid tumors (for review, see Yen 2003).

\section{Materials and methods}

Strains construction

$\Delta d d b 1$ was generated by transforming a wild-type strain with a linearized construct containing $\sim 500 \mathrm{bp}$ of $5^{\prime}$ - and $3^{\prime}$-UTR on either side of the G418 resistance cassette for replacement of the entire $d d b 1$ open reading frame, and was used for all experiments except for the spontaneous mutation assays, where we used a ddb1-disrupted strain. Stable diploid mat1-P/mat1-Mc strains for synchronization of meiosis were generated by protoplast fusion essentially as described by Willer et al. (1995). In order to eliminate haploidization by chromosome loss caused by $\Delta \operatorname{csn} 1$ and $\Delta d d b 1$ (Mundt et al. 2002; Zolezzi et al. 2002), complementing ade6-M210/ade6-M216 allele pairs were included in all diploid strains.

\section{Test systems to measure mutation rates}

Mutation rates of the ade6 alleles 485 and (GTCC) ${ }_{2}-1399$ were determined by fluctuation tests as described (Kunz and Fleck 2001; Marti et al. 2003b). Allele 485 is a C-to-G transversion that reverts to $\mathrm{Ade}^{+}$via base substitutions (Fleck et al. 1999); $(\text { GTCC) })_{2}-1399$ is an insertion of a GTCC tetra nucleotide at an existing GTCC sequence at position 1399 of the ade6 gene (the ATG start codon is at position 875). The insertion causes a frameshift and a premature stop codon in ade6 and reverts to $\mathrm{Ade}^{+}$through 1-bp/4-bp net deletions or 2-bp/5-bp net insertions at or around the GTCC insertion (Marti et al. 2003b). Each fluctuation test included seven or nine cultures grown in $1.5 \mathrm{~mL}$ of YEL in the case of 485, and 15 cultures grown in $5 \mathrm{~mL}$ of YEL in the case of $(G T C C)_{2}-1399$. Mutation rates were determined by the method of the median or from the proportion of cultures without $\mathrm{Ade}^{+}$(Luria and Delbrück 1943; Lea and Coulson 1949). For each genetic background, fluctuation tests were carried out at least three times. The nature of $\mathrm{Ade}^{+}$revertants was determined by sequencing of single-stranded DNA derived from PCR fragments as described (Kunz and Fleck 2001; Mansour et al. 2001).

\section{Western blots}

Protein extracts were made by the TCA precipitation method (Caspari et al. 2000). Spd1 was detected using anti-Spd1 polyclonal antibodies awo-1 (Woollard et al. 1996) diluted 1:200. Spd1-TAP was detected on Western blots using HRP-coupled antibodies from rabbit (DakoCytomation, cat. \#P0161) diluted 1:3000. For loading control, we used anti-Tubulin monoclonal antibodies (Woods et al. 1989) diluted 1:3000. Primary antibodies were detected using HRP-coupled secondary antibodies diluted 1:5000. Blots were developed with ECL+ (for Spd1-TAP and Spd1) and with ECL (for Tubulin) from Amersham according to instructions.

\section{Induction of meiosis}

Synchronous meiosis was induced in the following way: Stable diploid mat1-P/mat1-Mc cells of the indicated genotype were grown in MSL medium (Egel et al. 1994) at $30^{\circ} \mathrm{C}$ to a density of $5 \times 10^{6}$ cells $/ \mathrm{mL}$, and then transferred at the same density to MSL without nitrogen and containing $1 \mu \mathrm{g} / \mathrm{mL}$ of synthetic Mfactor (supplied by Schafer-N) (Wang et al. 1994). A detailed account for this synchronization procedure will be published elsewhere.

\section{Flow cytometry}

Samples for flow cytometry were fixed and stained with propidium iodine as described by Pereira and Jones (2001) with the following modification: Prior to RNase treatment, the cells were incubated with $1 \mathrm{mg} / \mathrm{mL}$ of pepsin in $0.1 \mathrm{~N} \mathrm{HCl}$ for $1 \mathrm{~h}$ at 
room temperature. The cells were analyzed on a Beckton Dickinson FACScan.

\section{Nucleotide pool measurements}

For labeling, $200 \mu \mathrm{Ci}$ of ${ }^{32} \mathrm{P}$ was added to a $0.5-\mathrm{mL}$ culture containing $4 \times 10^{6}$ cells in MSL medium buffered with acetic acid and containing $50 \mu \mathrm{M}$ cold phosphate. After $5 \mathrm{~h}, 400 \mu \mathrm{L}$ was transferred to $50 \mu \mathrm{L}$ of prechilled $26.5 \mathrm{M}$ formic acid, followed by three freeze-thaw cycles. Cell debris was removed by centrifugation and $100 \mu \mathrm{L}$ spotted onto a polyethyleneimine plate to separate nucleotides in two dimensions as described (Jensen et al. 1979|. First-dimension thin-layer chromatography was developed in $0.85 \mathrm{M} \mathrm{KH}_{2} \mathrm{PO}_{4}$ adjusted to $\mathrm{pH} 3.4$ with equimolar $\mathrm{H}_{3} \mathrm{PO}_{4}$. Following chromatography, the plates were washed twice in $10 \%(\mathrm{wt} / \mathrm{vol})$ citrate and twice in water. The dried plates were developed in the second dimension in $0.75 \mathrm{M} \mathrm{LiCl} /$ $7.5 \% \mathrm{H}_{3} \mathrm{BO}_{3}$ adjusted to $\mathrm{pH} 6.8$ by addition of solid $\mathrm{LiOH}$. The plates were quantified using a Storm PhosphorImager, and the spots were indentified by addition of cold trinucleotides.

\section{Acknowledgments}

We thank Jacky Hayles for the generous gift of anti-Spd1 antibody, Kaj Frank Jensen and Michael Sørensen for sharing their expertise on nucleotide pools measurements, and Richard Egel for discussions and critical reading of the manuscript. This work was supported by the Danish Cancer Society (O.N.), CRUK (grant no. C5514 to A.M.C.), and a VELUX visiting professorship (O.F. to visit O.N.).

\section{References}

Aboussekhra, A., Biggerstaff, M., Shivji, M.K., Vilpo, J.A., Moncollin, V., Podust, V.N., Protic, M., Hubscher, U., Egly, J.M., and Wood, R.D. 1995. Mammalian DNA nucleotide excision repair reconstituted with purified protein components. Cell 80: 859-868.

Angus, S.P., Wheeler, L.J., Ranmal, S.A., Zhang, X., Markey, M.P., Mathews, C.K., and Knudsen, E.S. 2002. Retinoblastoma tumor suppressor targets dNTP metabolism to regulate DNA replication. J. Biol. Chem. 277: 44376-44384.

Bondar, T., Mirkin, E.V., Ucker, D.S., Walden, W.E., Mirkin, S.M., and Raychaudhuri, P. 2003. Schizosaccharomyces pombe $\mathrm{Ddb} 1$ is functionally linked to the replication checkpoint pathway. J. Biol. Chem. 278: 37006-37014.

Bondar, T., Ponomarev, A., and Raychaudhuri, P. 2004. Ddb1 is required for the proteolysis of the Schizosaccharomyces pombe replication inhibitor Spd1 during S phase and after DNA damage. J. Biol. Chem. 279: 9937-9943.

Caspari, T. and Carr, A.M. 1999. DNA structure checkpoint pathways in Schizosaccharomyces pombe. Biochimie 81: 173-181.

Caspari, T., Dahlen, M., Kanter-Smoler, G., Lindsay, H.D., Hofmann, K., Papadimitriou, K., Sunnerhagen, P., and Carr, A.M. 2000. Characterization of Schizosaccharomyces pombe Hus1: A PCNA-related protein that associates with Rad1 and Rad9. Mol. Cell. Biol. 20: 1254-1262.

Chabes, A., Georgieva, B., Domkin, V., Zhao, X., Rothstein, R., and Thelander, L. 2003. Survival of DNA damage in yeast directly depends on increased dNTP levels allowed by relaxed feedback inhibition of ribonucleotide reductase. Cell 112: 391-401.

Cope, G.A. and Deshaies, R.J. 2003. COP9 signalosome: A multifunctional regulator of SCF and other cullin-based ubiquitin ligases. Cell 114: 663-671.

Dichtl, B., Blank, D., Sadowski, M., Hubner, W., Weiser, S., and
Keller, W. 2002. Yhh1p/Cft1p directly links poly(A) site recognition and RNA polymerase II transcription termination. EMBO I. 21: 4125-4135.

Egel, R., Willer, M., Kjaerulff, S., Davey, J., and Nielsen, O. 1994. Assessment of pheromone production and response in fission yeast by a halo test of induced sporulation. Yeast 10: $1347-1354$.

Ekwall, K., Nimmo, E.R., Javerzat, J.P., Borgstrom, B., Egel, R., Cranston, G., and Allshire, R. 1996. Mutations in the fission yeast silencing factors $c l r 4^{+}$and rik $1^{+}$disrupt the localisation of the chromo domain protein Swi6p and impair centromere function. J. Cell. Sci. 109: 2637-2648.

Fantes, P. and Nurse, P. 1977. Control of cell size at division in fission yeast by a growth-modulated size control over nuclear division. Exp. Cell Res. 107: 377-386.

Fernandez-Sarabia, M.J., McInerny, C., Harris, P., Gordon, C., and Fantes, P. 1993. The cell cycle genes $c d c 22^{+}$and suc22 ${ }^{+}$ of the fission yeast Schizosaccharomyces pombe encode the large and small subunits of ribonucleotide reductase. Mol. Gen. Genet. 238: 241-251.

Fleck, O., Lehmann, E., Schar, P., and Kohli, J. 1999. Involvement of nucleotide-excision repair in msh2 pms1-independent mismatch repair. Nat. Genet. 21: 314-317.

Friedberg, E.C., Wagner, R., and Radman, M. 2002. Specialized DNA polymerases, cellular survival, and the genesis of mutations. Science 296: 1627-1630.

Grallert, B. and Sipiczki, M. 1991. Common genes and pathways in the regulation of the mitotic and meiotic cell cycles of Schizosaccharomyces pombe. Curr. Genet. 20: 199-204.

Groisman, R., Polanowska, J., Kuraoka, I., Sawada, J., Saijo, M., Drapkin, R., Kisselev, A.F., Tanaka, K., and Nakatani, Y. 2003. The ubiquitin ligase activity in the DDB2 and CSA complexes is differentially regulated by the COP9 signalosome in response to DNA damage. Cell 113: 357-367.

Hara, R., Mo, J., and Sancar, A. 2000. DNA damage in the nucleosome core is refractory to repair by human excision nuclease. Mol. Cell. Biol. 20: 9173-9181

Higa, L.A., Mihaylov, I.S., Banks, D.P., Zheng, J., and Zhang, H. 2003. Radiation-mediated proteolysis of CDT1 by CUL4ROC1 and CSN complexes constitutes a new checkpoint. Nat. Cell. Biol. 5: 1008-1015.

$\mathrm{Hu}$, J., McCall, C.M., Ohta, T., and Xiong, Y. 2004. Targeted ubiquitination of CDT1 by the DDB1-CUL4A-ROC1 ligase in response to DNA damage. Nat. Cell. Biol. 6: 1003-1009.

Huang, M. and Elledge, S.J. 1997. Identification of RNR4, encoding a second essential small subunit of ribonucleotide reductase in Saccharomyces cerevisiae. Mol. Cell. Biol. 17: 6105-6113.

Huang, M., Zhou, Z., and Elledge, S.J. 1998. The DNA replication and damage checkpoint pathways induce transcription by inhibition of the Crt1 repressor. Cell 94: 595-605.

Hubscher, U., Maga, G., and Spadari, S. 2002. Eukaryotic DNA polymerases. Annu. Rev. Biochem. 71: 133-163.

Itoh, T., O'Shea, C., and Linn, S. 2003. Impaired regulation of tumor suppressor p53 caused by mutations in the xeroderma pigmentosum $D D B 2$ gene: Mutual regulatory interactions between p48(DDB2) and p53. Mol. Cell. Biol. 23: 7540-7553.

Itoh, T., Cado, D., Kamide, R., and Linn, S. 2004. DDB2 gene disruption leads to skin tumors and resistance to apoptosis after exposure to ultraviolet light but not a chemical carcinogen. Proc. Natl. Acad. Sci. 101: 2052-2057.

Jensen, K.F., Houlberg, U., and Nygaard, P. 1979. Thin-layer chromatographic methods to isolate ${ }^{32} \mathrm{P}$-labeled 5-phosphoribosyl- $\alpha-1$-pyrophosphate (PRPP): Determination of cellular PRPP pools and assay of PRPP synthetase activity. Anal. Biochem. 98: 254-263. 
Kai, M. and Wang, T.S. 2003. Checkpoint activation regulates mutagenic translesion synthesis. Genes \& Dev. 17: 64-76.

Kunz, C. and Fleck, O. 2001. Role of the DNA repair nucleases Rad13, Rad2 and Uve1 of Schizosaccharomyces pombe in mismatch correction. J. Mol. Biol. 313: 241-253.

Laplaza, J.M., Bostick, M., Scholes, D.T., Curcio, M.J., and Callis, J. 2004. Saccharomyces cerevisiae ubiquitin-like protein Rub1 conjugates to cullin proteins Rtt101 and Cul3 in vivo. Biochem. J. 377: 459-467.

Lea, D.E. and Coulson, C.A. 1949. The distribution of the number of mutants in bacterial populations. J. Genet. 49: 264285.

Lehmann, A.R. 2002. Replication of damaged DNA in mammalian cells: New solutions to an old problem. Mutat. Res. 509: 23-34.

Liu, C., Powell, K.A., Mundt, K., Wu, L., Carr, A.M., and Caspari, T. 2003. Cop9/signalosome subunits and Pcu4 regulate ribonucleotide reductase by both checkpoint-dependent and -independent mechanisms. Genes \& Dev. 17: 1130-1140.

Luria, S.E. and Delbrück, M. 1943. Mutations of bacteria from virus sensitivity to virus resistance. Genetics 28: 491-511.

Lyapina, S., Cope, G., Shevchenko, A., Serino, G., Tsuge, T., Zhou, C., Wolf, D.A., Wei, N., and Deshaies, R.J. 2001. Promotion of NEDD-CUL1 conjugate cleavage by COP9 signalosome. Science 292: 1382-1385.

Mansour, A.A., Tornier, C., Lehmann, E., Darmon, M., and Fleck, O. 2001. Control of GT repeat stability in Schizosaccharomyces pombe by mismatch repair factors. Genetics 158: $77-85$.

Marti, T.M., Kunz, C., and Fleck, O. 2003a. Repair of damaged and mismatched DNA by the XPC homologues Rhp41 and Rhp42 of fission yeast. Genetics 164: 457-467.

Marti, T.M., Mansour, A.A., Lehmann, E., and Fleck, O. 2003b. Different frameshift mutation spectra in non-repetitive DNA of MutS $\alpha$ - and MutL $\alpha$-deficient fission yeast cells. DNA Repair 2: 571-580.

Mu, D., Park, C.H., Matsunaga, T., Hsu, D.S., Reardon, J.T., and Sancar, A. 1995. Reconstitution of human DNA repair excision nuclease in a highly defined system. J. Biol. Chem. 270: $2415-2418$.

Mundt, K.E., Porte, J., Murray, J.M., Brikos, C., Christensen, P.U., Caspari, T., Hagan, I.M., Millar, J.B., Simanis, V., Hofmann, K., et al. 1999. The COP9/signalosome complex is conserved in fission yeast and has a role in S phase. Curr. Biol. 9: 1427-1430.

Mundt, K.E., Liu, C., and Carr, A.M. 2002. Deletion mutants in COP9/signalosome subunits in fission yeast Schizosaccharomyces pombe display distinct phenotypes. Mol. Biol. Cell 13: 493-502.

Nakayama, J., Rice, J.C., Strahl, B.D., Allis, C.D., and Grewal, S.I. 2001. Role of histone H3 lysine 9 methylation in epigenetic control of heterochromatin assembly. Science 292: 110-113.

Neuwald, A.F. and Poleksic, A. 2000. PSI-BLAST searches using hidden markov models of structural repeats: Prediction of an unusual sliding DNA clamp and of $\beta$-propellers in UV-damaged DNA-binding protein. Nucleic Acids. Res. 28: 35703580.

Nielsen, O. 2004. Mating-type control and differentiation. In The molecular biology of Schizosaccharomyces pombe (ed. R. Egel), pp. 281-296. Springer-Verlag Berlin, Heidelberg, Germany.

Pereira, P.S. and Jones, N.C. 2001. The RGS domain-containing fission yeast protein, Rgs $1 \mathrm{p}$, regulates pheromone signalling and is required for mating. Genes Cells 6: 789-802.

Rödel, C., Kirchhoff, S., and Schmidt, H. 1992. The protein se- quence and some intron positions are conserved between the switching gene swilo of Schizosaccharomyces pombe and the human excision repair gene ERCC1. Nucleic Acids Res. 20: 6347-6353.

Rudolph, C., Kunz, C., Parisi, S., Lehmann, E., Hartsuiker, E., Fartmann, B., Kramer, W., Kohli, J., and Fleck, O. 1999. The msh2 gene of Schizosaccharomyces pombe is involved in mismatch repair, mating-type switching, and meiotic chromosome organization. Mol. Cell. Biol. 19: 241-250.

Stern, B. and Nurse, P. 1997. Fission yeast pheromone blocks S-phase by inhibiting the G1 cyclin B-p34cdc2 kinase. EMBO J. 16: 534-544.

Svejstrup, J.Q. 2002. Mechanisms of transcription-coupled DNA repair. Nat. Rev. Mol. Cell Biol. 3: 21-29.

Tang, J. and Chu, G. 2002. Xeroderma pigmentosum complementation group E and UV-damaged DNA-binding protein. DNA Repair 1: 601-616.

Tuzon, C.T., Borgstrom, B., Weilguny, D., Egel, R., Cooper, J.P., and Nielsen, O. 2004. The fission yeast heterochromatin protein Rik1 is required for telomere clustering during meiosis. J. Cell Biol. 165: 759-765.

Wang, Z. 2001. Translesion synthesis by the UmuC family of DNA polymerases. Mutat. Res. 486: 59-70.

Wang, S.H., Xue, C.B., Nielsen, O., Davey, J., and Naider, F. 1994. Chemical synthesis of the M-factor mating phero mone from Schizosaccharomyces pombe. Yeast 10: 595-601.

Wei, N. and Deng, X.W. 2003. The COP9 signalosome. Annu. Rev. Cell. Dev. Biol. 19: 261-286.

Willer, M., Hoffmann, L., Styrkarsdottir, U., Egel, R., Davey, J., and Nielsen, O. 1995. Two-step activation of meiosis by the matl locus in Schizosaccharomyces pombe. Mol. Cell. Biol. 15: 4964-4970.

Wittschieben, B.B. and Wood, R.D. 2003. DDB complexities. DNA Repair 2: 1065-1069.

Woods, A., Sherwin, T., Sasse, R., MacRae, T.H., Baines, A.J., and Gull, K. 1989. Definition of individual components within the cytoskeleton of Trypanosoma brucei by a library of monoclonal antibodies. J. Cell. Sci. 93: 491-500.

Woollard, A., Basi, G., and Nurse, P. 1996. A novel S phase inhibitor in fission yeast. EMBO J. 15: 4603-4612.

Yamamoto, M. 2004. Initiation of meiosis. In The molecular biology of Schizosaccharomyces pombe (ed. R. Egel), pp. 297-309. Springer-Verlag Berlin, Heidelberg, Germany.

Yen, Y. 2003. Ribonucleotide reductase subunit one as gene therapy target: Commentary re: M-Y. Cao et al., Adenovirusmediated ribonucleotide reductase R1 gene therapy of human colon adenocarcinoma. Clin. Cancer Res. 9: 4304-4308. Clin. Cancer Res. 9: 4304-4308.

Zhao, X., Muller, E.G., and Rothstein, R. 1998. A suppressor of two essential checkpoint genes identifies a novel protein that negatively affects dNTP pools. Mol. Cell 2: 329-340.

Zhong, W., Feng, H., Santiago, F.E., and Kipreos, E.T. 2003. CUL-4 ubiquitin ligase maintains genome stability by restraining DNA-replication licensing. Nature 423: 885-889.

Zhou, C., Seibert, V., Geyer, R., Rhee, E., Lyapina, S., Cope, G., Deshaies, R.J., and Wolf, D.A. 2001. The fission yeast COP9/ signalosome is involved in cullin modification by ubiquitinrelated Ned8p. BMC Biochem. 2: 7.

Zhou, C., Wee, S., Rhee, E., Naumann, M., Dubiel, W., and Wolf, D.A. 2003. Fission yeast COP9/signalosome suppresses cullin activity through recruitment of the deubiquitylating enzyme Ubp12p. Mol. Cell 11: 927-938.

Zolezzi, F., Fuss, J., Uzawa, S., and Linn, S. 2002. Characterization of a Schizosaccharomyces pombe strain deleted for a sequence homologue of the human damaged DNA binding 1 (DDB1) gene. J. Biol. Chem. 277: 41183-41191. 


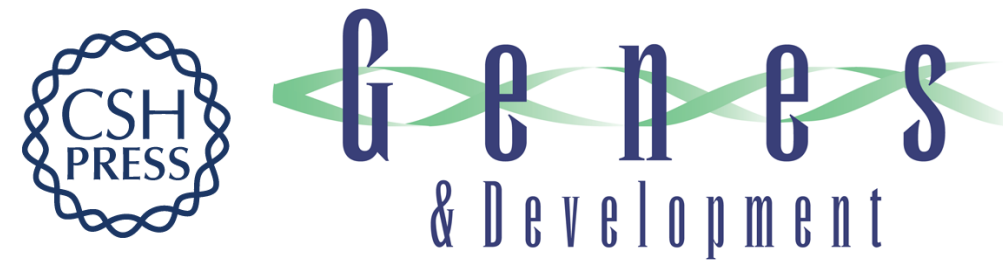

\section{Ddb1 controls genome stability and meiosis in fission yeast}

Christian Holmberg, Oliver Fleck, Heidi A. Hansen, et al.

Genes Dev. 2005, 19:

Access the most recent version at doi:10.1101/gad.329905

Supplemental

Material

References

License

Email Alerting Service
http://genesdev.cshlp.org/content/suppl/2005/04/05/19.7.853.DC1

This article cites 62 articles, 27 of which can be accessed free at: http://genesdev.cshlp.org/content/19/7/853.full.html\#ref-list-1

Receive free email alerts when new articles cite this article - sign up in the box at the top right corner of the article or click here.

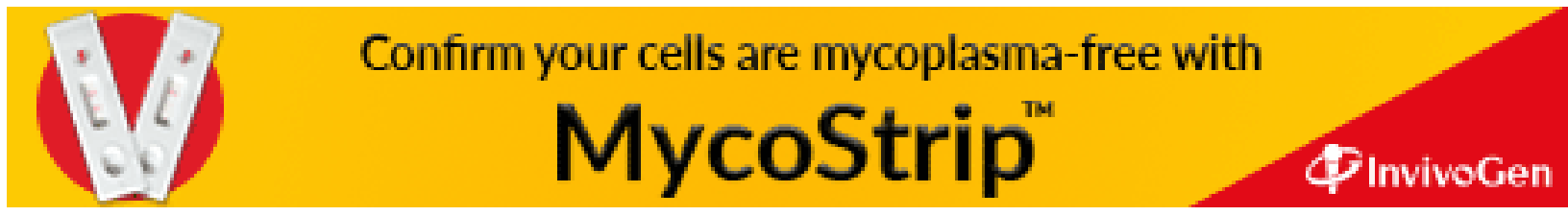

